\title{
Study on Animal Model Characterization of Chinese Medicine
}

\author{
Xu ZHANG, Hui ZHOU, Xuan-xuan ZHU, Yi LIANG, Jia-chen \\ WANG $^{2}$, Yan-yan MIAO ${ }^{2}$ and Ming-san MIAO* \\ Henan University of Traditional Chinese Medicine, Zhengzhou, HENAN,CHAIN
}

${ }^{*}$ Corresponding author

Keywords: Representation, Animal model of Chinese medicine, Evaluation standard.

\begin{abstract}
Characterization can reflect objectivity and represent objective things. With the development of pharmacology of Chinese Medicine in recent years and the deepening of animal model research in Chinese medicine, how to accurately and quickly copy the successful model animals according to the characterization has become a new direction to be studied urgently. In this paper, it includes the meaning of the method of characterization and the status quo of the research, the application of the method of characterization in the animal model of Chinese medicine, and the evaluation and evaluation of the value and prospect of the evaluation of animal model in the study. We refers to an accurate, intuitive and convenient Of the Chinese animal model evaluation method to establish a certain theoretical basis. It aims to establish an accurate, intuitive and convenient method for the evaluation of animal model to provide a theoretical basis.
\end{abstract}

\section{Introduction}

Characterization, also known as knowledge representation, is intended to be one of the core concepts of cognitive psychology. It can reflect the objective things, and be on behalf of objective things. With the development of pharmacology of Chinese Medicine in recent years and the deepening of animal model research in Chinese medicine, how to accurately and quickly model the successful model animals according to the feature representation has become a new direction to be studied urgently.

In this paper, We will talk about the meaning of the method of characterization and the status quo of the research, the application of the method of characterization in the animal model of Chinese medicine, and the evaluation and evaluation of the value and prospect of the evaluation of animal animal model in the study. It aims to establish a certain theoretical basis, so that we could establishan accurate, intuitive and convenient Of the Chinese animal model evaluation method.

\section{The Meaning of the Method of Characterization and the Status Quo of the Research}

The term "characterization" in this context refers to the characteristic manifestation of animal appearance. "governing exterior to infer interior" and "holism" is the core content of Chinese Medicine diagnosis, emphasizing the human body is a complete whole. So under the pathological state, it can be achieved by external symptoms to infer the internal organs of the pathological changes. The essence of the method is using the characterization analysis. Human and nature are a whole, and, in pathological conditions, different models of animals will show different animal characterization, for example, some used streptozotocin to replicate SD rats diabetes 
model[1], the normal group of rats hair luster , and it is normal, and the activities of them are normal, responsive. Compared with the blank group rats, the model group were diabetic typical of more than drink polyuria and weight loss symptoms, accompanied by apathetic, unresponsive and sensitive, Hair color and other symptoms; studies have shown that the model of benign prostatic hyperplasia model can occur less typical symptoms of urine, so we can simply observe the cleanliness of the litter to determine the modeling situation[2]; There are different methods of rat Menopausal syndrome model[3], which collectively characterized by weight loss, in addition, perimenopausal syndrome, depressive symptoms such as depression, decreased the number of activities, decreased sugar consumption, perimenopausal syndrome, insomnia Sexual characteristics of the circadian rhythm and sleep disorders, etc .; characterization evaluation is also used for cerebrovascular disease animal model of the application of neurological function score is an important indicator of cerebral ischemia animal model evaluation, the current use of ZeaLonga Five grading standards[4]; cyclophosphamide-induced immunosuppressive mice in the injection of cyclophosphamide 3 days after the emergence of weight loss, curl, closed eyes and other symptoms, and normal mice were significantly different[5]. In addition, the characterization evaluation is also widely used in behavioral abnormal animal model evaluation. Characterization index is the most intuitive model evaluation index, therefore, in the process of screening the animal model, according to the changes in characterization, effective and efficient screening of the model replication of successful animals.

\section{The Application of the Method of Characterization in the Animal Model of Chinese Medicine}

In the animal experimental study, under the standard feeding environment, the normal animal hair is smooth and shiny, coat color is normal, rhythmic behavior, moderate drinking water, weight is relatively constant, less volatile, or with the animal Age increased and showed an increasing trend. In the pathological state, in addition to changes in biochemical indicators, the characterization of the characteristics of the animals' obvious changes will occur. In terms of the degree of change in the index, the following words are commonly used in existing animal experiment scientific papers[6] $-[25]$.

Table 1.The evaluation site of animal model and lesion status

\begin{tabular}{|l|l|l|}
\hline \multicolumn{2}{|c|}{ Evaluation site } & \multicolumn{1}{c|}{ Lesion state } \\
\hline \multirow{2}{*}{$\begin{array}{l}\text { overa } \\
\text { 11 }\end{array}$} & Coat color & Coat color as normal group; coat color yellow; hair less moist; \\
\cline { 2 - 3 } & Hair & $\begin{array}{l}\text { Hairy and dull, hairy luster; hair fluffy; supple and shiny; hair sticky } \\
\text { dull; hair dull and dull; vertical Hair; serious hair loss; dorsal fur is not } \\
\text { bright; hair removal }\end{array}$ \\
\cline { 2 - 4 } & $\begin{array}{l}\text { Bodytemp } \\
\text { eratu-re }\end{array}$ & $\begin{array}{l}\text { Skin cooler; body temperature drop; body temperature decreased; body } \\
\text { temperature dropped significantly; body temperature increased }\end{array}$ \\
\cline { 2 - 4 } behavior & $\begin{array}{l}\text { Reduce the number of hair loss, lack of activity, reduce the diet; reduce } \\
\text { the diet; trembling, bow; body weight loss; decreased mobility; diet } \\
\text { Lack of activity; slow activity; curl less moving; slightly reduced } \\
\text { appetite; slow growth and development; difficult to grasp; grasp the } \\
\text { grip when the amplitude of the change in the amount of disease; Small; } \\
\text { do not love sports }\end{array}$ \\
\cline { 2 - 3 } $\begin{array}{l}\text { body } \\
\text { weight }\end{array}$ & $\begin{array}{l}\text { Bodyweight decreased; body weight decreased; body weight decreased; } \\
\text { body mass showed steady growth, body weight decreased; body weight } \\
\text { decreased; }\end{array}$ \\
\hline \multicolumn{2}{|c|}{ Evaluation site } & \multicolumn{1}{c}{ Lesion state } \\
\hline
\end{tabular}




\begin{tabular}{|c|c|c|}
\hline & Excreta & $\begin{array}{l}\text { Fecal dry; oliguria and stool dry; urine volume reduction; moderate } \\
\text { urine output (litter than dry); fecal brown granules; significantly } \\
\text { increased urine output (litter wet); diarrhea; perianal filth ; Fecal } \\
\text { formation, soft; fecal loose pond, some not formed; fecal loose pond, } \\
\text { completely unformed; normal stool; stool thin }\end{array}$ \\
\hline & Sentiment & $\begin{array}{l}\text { Mental distress; irritability; lack of mental; poor mental state; mental } \\
\text { burnout; irritability; irritation was evasive response; look dementia, } \\
\text { depressed }\end{array}$ \\
\hline & Sleep & $\begin{array}{l}\text { Sleep disorders; circadian rhythm disappeared; circadian rhythm } \\
\text { disappeared during the day also non-stop; sleep time shortened, easy to } \\
\text { wake up. Sleep is gone; sleep is reduced }\end{array}$ \\
\hline \multirow[t]{4}{*}{$\begin{array}{l}\text { Partia } \\
1\end{array}$} & eye & $\begin{array}{l}\text { Eyelid ptosis; corneal opacity; eyeballs; cataract; eyebrow dark } \\
\text { red; eyeball red; eye dark red; eye dark red }\end{array}$ \\
\hline & skin & $\begin{array}{l}\text { Skin whitening, skin redness, mild swelling, there are obvious } \\
\text { folds, subcutaneous red blood spot scattered, there is a bubble uplift, a } \\
\text { large area }\end{array}$ \\
\hline & Breast & $\begin{array}{l}\text { Breast slightly elevated, nipple swelling increased; breast diameter } \\
\text { increased significantly, breast tissue was significantly proliferated; } \\
\text { breast tissue hyperplasia, breast diameter is still greater than the } \\
\text { administration group }\end{array}$ \\
\hline & Tongue & $\begin{array}{l}\text { Tongue reddish, tongue slightly green; tongue dark red partial } \\
\text { purple, tongue color dark purple; tongue bluish with ecchymosis; red } \\
\text { tongue }\end{array}$ \\
\hline
\end{tabular}

Table 1, cont. The evaluation site of animal model and lesion status

From the table 1 and Table 1, cont. Evaluation of characterization in animal model evaluation has a certain application. And the current mature animal model also has a unique behavioral scoring criteria, such as the use of ZeaLonga Five grading scale in cerebral ischemic animal models ${ }^{[21]}$; It could use BBB score score to evaluate the rat spinal cord injury model ${ }^{[22]}$;Laboratories on the skin disease model have sets of independent behavioral evaluation criteria, such as sore model score criteria ${ }^{[23]}$, body ringworm symptom score standard ${ }^{[24]}$. And for articular inflammatory diseases, behavioral disorders ${ }^{[25]}$ and the like, the specific characterization can be scored by the instrument.

\section{The Evaluation and Evaluation of the Value and Prospect of the Evaluation of Animal Model}

Presently, there are some progresses in the research on the evaluation of the animal model. At present, two kinds of research ideas ${ }^{[26]}$ are proposed, which are based on the characteristics of Chinese medicine clinical disease, As well as the "four diagnostic methods" reverse dialectical thinking model research, and at present, some scholars have successfully established rats, mice, miniature pigs and other illness combination model, involving the symptoms, including yin deficiency syndrome, yang deficiency syndrome, syndrome of blood stasis, dampness-phlegm syndrome, syndrome of qi deficiency, syndrome of stagnation of liver qi and spleen deficiency and so on. However, there are still some problems in the evaluation of the existing indicators and the terminology analysis described in the above literature. (1) At present, there is no in-depth study on the method of characterization for characterization analysis. (2) The application of apparent indication evaluation in animal experiment and animal model evaluation is not based on uniform and systematic criteria. In addition to individual research, animal model has a set of grading evaluation methods or individual laboratory The subjective degree is mainly based on the subjective level of the experimenter, and the result is not conducive to the development of the apparent 
indication evaluation. (3) In recent years, the research on the evaluation of animal model of Chinese medicine began to appear sporadically, but there is not yet a complete evaluation system of animal model.

Animal model characterization is very meaningful, which can be reflected from the overall level of animal body pathology. Therefore, the current research on animal model characterization index evaluation system should be meticulous, in-depth and systematic research. The key to the research of animal model characterization is how to set up data for the collection of characterization indicators, how to unify the norms and standards of evaluation, and how to eliminate human error, etc..Be committed to overcome these problems should be the future of Chinese medicine animal model characterization evaluation of the focus. Therefore, it is possible to establish a set of quantitative or semi-quantitative grading evaluation system, but also to develop the instrument and equipment which can be used to characterize the evaluation of indicators. This will help to quantify the grading evaluation of the index, and provide the basis for the application of the evaluation method. The application of this quick and easy method to evaluate the evaluation of animal models and animal experiments.

\section{Acknowledgments}

The research work was supported by National Natural Science Foundation of China (81274154) Zhongyuan scholar (162101510003) and Henan Province outstanding scientific and technological innovation team (TCJ2014-391).

\section{References}

[1] Effects of Modified Kaixin Powder on Learning and Memory in Dementia Mice with Multifactorial Neurological Damage [J]. Chinese Journal of Integrative Medicine on Cardio / Cerebrovascular Disease, Chinese Academy of Sciences, Beijing 100083, China. Journal of the University, 2017, (01): 44-48.

[2] Effects of Cholic Acid Components in Bovine Beef on Cerebral Ischemia Reperfusion Injury and Regulation in Rats [J]. Chinese Journal of Integrative Medicine on Cardio / Cerebrovascular Disease, Chinese Academy of Medical Sciences Comparison of stress in network [J]. Chinese Journal of Pharmacoepidemiology, 2017, (01): 11-19.

[3] Li Zhaoyi, Lu Min, Liang Wei, Liang Nengtang, Qin Xiaoyun, Xia Chunbo, Wang Junfeng, Chen Chen. Establishment and evaluation of diabetic rat model [J]. Chinese Journal of Primary Medicine, 2017, (01): 4-5.

[4] Wang Rui, Wang Qi, Jin Mingshun, Chen Xue, Wang Wei, Zhang Ning, Yang Dezhu, Liu Jianhua, Wang Guozhong, Liu Jicheng. Effects of resveratrol on the behavior of perimenopausal depression model mice and its mechanism [ $\mathrm{J}$ Chinese Journal of Experimental Chinese Medicine, 2017 (04): 132-139.

[5] FANG Xiao-yan, WANG Lin-lin, ZHANG Fan, SUN Jian-ning, MIAO Ming-san. Protective effects of cerebral ischemic preconditioning on cerebralischemia-reperfusion in rats and expression of neurotrophic factors [J]. Chinese Journal of Experimental Traditional Medical Formulas, 2016 (18): 112-117.

[6] ShiJing-jing, Shi Bo, Miao Ming three, Li Qingya. Fuzheng detox tablets on hydrocortisone-induced immunosuppressive mice peripheral blood $\mathrm{T}$ lymphocytes 
and immune organs [J]. World Science and Technology - Chinese Medicine, 2016 (03): 457-463.

[7] MIAO Ming-san, CUI Yu-sheng, XI Peng, CHEN Yuan-peng. Effects of different varieties of bitter almond oil paste on sore rats $[\mathrm{J}]$.Chinese Journal of Chinese Medicine, 2016, (03): 821-824.

[8] Li Miehui, Wu Xiao, Wei Ying, Dong Jingcheng. Establishment and behavioral evaluation of depression-induced model of C57BL / 6 mice induced by social stress [J]. Zhongguo Zhong Xi Yi Jie Za Zhi, 2016, (02): 229- 233.

[J] Chinese Journal of Chinese Medicine, 2016 (02): 392-395. [J] .Chinese Journal of Chinese Medicine, 2016 (02): 392-395.

[J] Chinese Journal of Chinese Medicine, 2016, (01): 67-70. [J]. Chinese Journal of Chinese Medicine, 2016 (01): 67-70. [J]. Chinese Journal of Chinese Medicine, 2016 (01): 67-70.

[11] Shi Jingjing, Shi Bo, Miao Ming three. Astragalus polysaccharide on cyclophosphamide induced immunosuppressive mice immune function $[\mathrm{J}]$. Chinese Journal of Chinese Medicine, 2016, (02): 243-246.

[12] Guo Lin, Miao Ming three. Based on the characteristics of clinical manifestations of benign prostatic hyperplasia animal model analysis $[\mathrm{J}]$. Chinese Journal of Chinese Medicine, 2016, (01): 261-264.

[13] Chen Zhang Min, Li Rongke, Zhang Pengfei, Wan Shengfang, Zhang Lingwei, Guo Yulong, Wang Qiulan. Effect of Angelica sinensis - Hongqi Ultrafiltration Membrane Extract on Expression of TGF- $\beta-1$ in Renal Tissue of Diabetic Nephropathy Rats [J]. Chinese Journal of Chinese Medicine Journal, 2017, (05): 113-118.

[14]Zhang Fan, Xu Shan, Liu Pengpeng, Jia Tianzhu. Huang Bai different processed products on hyperthyroidism kidney yin deficiency model rats thyroid and adrenal cortical function [J]. Chinese pharmacy, 2017, (01): 27-30.

[15] Li Jie, Zhang Lishan, Yu Weixia, Zhou Shaoyong, Wang Xinyang, Wei Xinxin. Effects of Qiangqin Zhushou Decoction on Pulmonary Tissue Transport Capacity of Penicillin in Rats with Acute Exacerbation of Chronic Obstructive Pulmonary Disease [J]. Chinese Journal of Chinese Medicine, 2017, (01): 254-259.

[16] Yang Mingyue, Yue Xiaoli, Zhou Min, Liu Haokun, Yao Yao, Xuan Lihua, Wang Yunlai, Xu vanadium. Based on the electrolyte study of Danggui Shaoyao San on nephrotic syndrome in rats [J]Journal of Liaoning University of Chinese Medicine, (01): 39-41.[J] Anti-infectivePharmacy, 2016, (05): 966-969 [J]. Journal of Infectious Diseases, Chinese Academy of Sciences, Beijing 100080, China.

[18] Long Si-min, Wang Qun, Wang Xiaoping, Cai Huicong, Wang Shan-shan, Zhang $\mathrm{Yu}$. Effects of lobular hyperplasia on the pathology of mammary gland in mammary gland hyperplasia [J] .Chinese Journal of Chinese Medicine, 2016 (16): 30-32 The

[19] Wang Zigao, Yang Yasong, Xu Yixuan, Zu Hengbing. Effects of Tempol on Cerebral Cerebral Vascular Disease in Stroke-prone Spontaneously Hypertensive Model Rats [J]. Chinese Journal of Neurosurgery, 2016, (03): 288 -292. 
[20] Wu Jianhui, Xu Sichong, Pan Qi, Su Xin, Yan Han, Li Lei, Shao Yue, Sun Zuyue. Comparison of spontaneous and testosterone-induced benign prostatic hyperplasia model [J]. Chinese Journal of Experimental Animal Science, 2013, ): $21-26+94$.

[21]LU Chang, DU Xiao, HE Xiao-li, WANG Yue-hua, DU Guan-hua. Study on the neuroprotective effect of Xiao Duxiu Decoction on early recovery of focal cerebral ischemia / reperfusion in rats [J]. Chinese Journal of Pharmacology, 2016, (07): 938-944.

[22]CHENG Su-li, XU Jia-chun, WANG Jian-song, JIAO Zhao-hua, ZHOU Zhen, SUN Li-ming, LI Yan.Improvement and behavior evaluation of Allen's modeling method in acute spinal cord injury rats $[\mathrm{J}]$.Chinese Journal of Chinese Medicine, 2015, (10): 43-45 The

[23]Tian Shuo, Bai Ming, Miao Ming three. Based on perimenopausal syndrome clinical characteristics of the animal model analysis [J]. Chinese Journal of Experimental Prescription, 2015, (19): 217-221.

[24] Yang Dian-hui, Wang Jian, Han Jing, Liu Yingying. Migraine model of behavioral evaluation method [J]. Liaoning Journal of Chinese Medicine, 2015, (03): 650-652.

[25]Zhao Gui-zhi, Wang Xuping, Huang Xiao-wen, Yang Ming-yan, Shou Dan. Study on the model and behavior evaluation of common dementia rats with common carotid artery ligation combined with galactose injection [J]. Chinese Medical Journal, 2014, (04): 34 -37.

[26]Lin Zhi-jian, Zhang Bing, Liu Xiao-qing, Zhu Wen-jing. Chinese medicine syndrome model evaluation study [J]. Chinese Journal of Chinese Medicine, 2013, (08): 2217-2221. 